\title{
ON THE NON-UNIQUENESS OF ELASTIC ROTATIONS FOR DEFORMATIONS OF MATERIALS WITH ELASTIC RANGE
}

\author{
By DAVID R. OWEN (Carnegie-Mellon University)
}

\begin{abstract}
In this note precise definitions of the concepts of elastic and permanent deformation are used to establish non-uniqueness of elastic rotations for deformations of materials with elastic range.

1. Introduction. A feature common to theories of elastic-plastic materials is a representation of deformations in terms of elastic and inelastic parts. The question of the uniqueness of such a representation has been commented upon by many authors. In [1], [2], and [3], the inherent non-uniqueness of such representations has been asserted. On the other hand, some writers (see [4], for example) have assumed uniqueness of such representations. The purpose of this note is to state precise conditions under which non-uniqueness (in the form of non-uniqueness of elastic rotations) arises in a mechanical theory proposed by the present author [1]. Roughly speaking, I show that, in a material with elastic range, the larger the symmetry group the larger the number of possibilities for the elastic rotation. In particular, the elastic rotation is shown to be arbitrary for isotropic materials.
\end{abstract}

2. Basic concepts. In this section, I summarize the concepts and results presented in [1], as well as new results due to Kratochvil and Silhavy, which are relevant to the discussion which follows.

A material with elastic range is a material whose stress response

$$
T(t)=\Pi\left(F^{t}\right)
$$

(with $T(t)$ the current stress tensor and $F^{t}$ the history of deformation gradient up to time $t$ ) satisfies the additional restriction that continuations of the deformation gradient history $F^{t}$ which remain in a set $\mathbf{E}\left(F^{t}\right)$ produce a path-independent response. Accordingly, one can write

$$
\Pi\left(G^{\tau}\right)=\Pi^{*}\left(A, F^{t}\right)
$$

where $G^{\tau}$ is a continuation of $F^{t}$ which remains in $\mathrm{E}\left(F^{t}\right)$ and ends at the point $A$ in $\mathbf{E}\left(F^{t}\right)$. The set $\mathbf{E}\left(F^{t}\right)$ is called the elastic range corresponding to $F^{t}$ and the function $\Pi^{*}\left(\cdot, F^{t}\right)$ is called the elastic response determined by $F^{t}$ (see $[1$, p. 88]).

A proper orthogonal tensor $Q_{0}$ is called a symmetry transformation for a material with elastic range if the condition

$$
\Pi\left(F^{t} Q_{0}\right)=\Pi\left(F^{t}\right)
$$

holds for all histories $F^{t}$. The symmetry transformations for a given material form a group under multiplication of tensors; therefore, $Q_{0}$ is a symmetry transformation if and only if $Q_{0}{ }^{T}$ is a symmetry transformation. 
The response functional $\Pi$ depends, of course, upon a specified reference configuration with respect to which deformation histories $F^{t}$ are computed from given motions of a body. The particular history $1^{\dagger}$, which corresponds to rest in the reference configuration, here is assumed to obey the following conditions:

$(\beta 2)$ for each tensor $A$ and symmetry transformation $Q_{0}$ such that $A \in \mathbf{E}\left(1^{\dagger}\right) \cap$ $\mathbf{E}\left(Q_{0}^{\dagger}\right), \Pi^{*}\left(A, Q_{0}^{\dagger}\right)=\Pi^{*}\left(A, 1^{\dagger}\right)$

(B3) for each symmetry transformation $Q_{0}, \mathbf{E}\left(1^{\dagger}\right) Q_{0} \subset \mathbf{E}\left(1^{\dagger}\right)$.

Here, $Q_{0}{ }^{\dagger}$ is the constant history with value $Q_{0}$. Condition ( $\left.\beta 2\right)$ tells us that states of rest in the reference configuration and in a configuration obtained by a symmetry transformation of the reference configuration yield identical elastic responses. Condition ( $\beta 3)$ says, in suggestive terms, that symmetry transformations applied to states of strain in the initial elastic range produce states of strain which again are in the initial elastic range. These conditions are closely related to conditions $(\alpha 2)$ and $(\alpha 3)$ in the definition of "annealed state" given in [1, p. 90]. It should be noted that only symmetry transformations $Q_{0}$ enter in the statements of $(\beta 2)$ and $(\beta 3)$, whereas $(\alpha 2)$ and $(\alpha 3)$ involve in general a larger class of tensors, and $(\beta 2)$ and $(\beta 3)$ thus are weaker restrictions than $(\alpha 2)$ and $(\alpha 3)$.

The "principle of material frame indifference" $([5$, p. 44]) here tells us that $\Pi$ satisfies the condition

$$
\Pi\left(Q^{t} F^{t}\right)=Q(t) \Pi\left(F^{t}\right) Q(t)^{T}
$$

for every history $F^{t}$ and orthogonal valued history $Q^{t}$. In a private communication to me, Kratochvil and Silhavy have proved that this identity implies for materials with elastic range that

$$
Q \mathbf{E}\left(F^{t}\right)=\mathbf{E}\left(F^{t}\right)=\mathbf{E}\left(Q^{t} F\right)
$$

for every proper orthogonal tensor $Q$ and orthogonal valued history $Q^{t}$. I shall use these results in the subsequent development, as they allow me to develop the present ideas for a broader class of materials than I had previously considered [6].

Given a history $F^{t}$, a second history $F_{p}{ }^{t}$ is said to be a permanent deformation history corresponding to $F^{t}$ if the following conditions hold:

(P1) $F_{p}{ }^{t}(\sigma)$ is in the elastic range determined by $F^{t-\sigma}$, for every $\sigma \geq 0\left(F_{p}{ }^{t}(\sigma)\right.$ represents the permanent deformation at time $t-\sigma$ );

(P2) as $\sigma$ tends to zero, the points $F_{p}{ }^{t}(\sigma)$ are bounded away from the boundary $\partial \mathbf{E}\left(F^{t}\right)$ of the elastic range $\mathbf{E}\left(F^{t}\right)$;

(P3) a fixed function $\Pi_{0}$ determines the elastic response through the relation $\Pi^{*}\left(A, F^{t-\sigma}\right)=\Pi_{0}\left(A\left(F_{p}{ }^{t}(\sigma)\right)^{-1}\right)$ for every $\sigma \geq 0$ and every $A$ in $\mathbf{E}\left(F^{t-\sigma}\right)$ sufficiently close to $F_{p}{ }^{t}(\sigma)$.

It should be noted that Condition (P3) embodies the assumption that only the current permanent deformation and the current total deformation are necessary for the determination of the current stress at points in the current elastic range which are near the current permanent deformation. In (P3), $\left(F_{p}{ }^{t}(\sigma)\right)^{-1}$ denotes the inverse of $F_{p}{ }^{t}(\sigma)$.

I assume in the following development that the history $1^{\dagger}$, corresponding to rest in the given reference configuration, is a permanent deformation history corresponding to itself (see [1, A3]). 
3. Non-uniqueness of permanent deformations. As the main step toward establishing the non-uniqueness of elastic rotations, a result is presented below which gives conditions under which permanent deformations are not unique. Before giving this result, a preliminary remark is needed.

If a proper orthogonal tensor $Q_{0}$ is a symmetry transformation for a material with elastic range, then the same tensor is an element of the symmetry group of the function $\Pi_{0}$, i.e. the condition $\Pi_{0}\left(A Q_{0}\right)=\Pi_{0}(A)$ holds for all tensors $A$ (sufficiently near the identity tensor) whenever the condition $\Pi\left(F^{t} Q_{0}\right)=\Pi\left(F^{t}\right)$ holds for all histories $F^{t}$.

To prove this remark, we let $A$ be in $\mathbf{E}\left(1^{\dagger}\right)$. Since $1^{\dagger}$ is, by hypothesis, a permanent deformation history corresponding to itself, we can apply (P3), with $F^{t}=1^{\dagger}, F_{p}{ }^{t}=1^{\dagger}$ and $A$ close to 1 , to write

$$
\Pi_{0}(A)=\Pi_{0}\left(A 1^{-1}\right)=\Pi^{*}\left(A, 1^{\dagger}\right) .
$$

Moreover, for each orthogonal tensor $Q$, the relations $\left(^{*}\right)$, with $F^{t}=1^{\dagger}$ and $Q^{t}=Q^{\dagger}$, here yield $\mathbf{E}\left(1^{\dagger}\right)=\mathbf{E}\left(Q^{\dagger}\right)$, and we may conclude that the tensor $A$ also is in $\mathbf{E}\left(Q^{\dagger}\right)$. If we let $Q=Q_{0}$ be a symmetry transformation, then, in view of ( $\left.\beta 2\right)$, the previous equation for $\Pi_{0}$ implies

$$
\Pi_{0}(A)=\Pi^{*}\left(A, 1^{\dagger}\right)=\Pi^{*}\left(A, Q_{0}^{\dagger}\right) .
$$

Now, $\Pi^{*}\left(A, Q_{0}{ }^{\dagger}\right)$ represents the stress due to a continuation $G^{r}$ of $Q_{0}{ }^{\dagger}$ which remains in $\mathbf{E}\left(Q_{0}{ }^{\dagger}\right)$ and obeys $G^{\tau}(0)=A$. Therefore, the history $G^{r} Q_{0}{ }^{T}$ represents a continuation of $1^{\dagger}$ which remains in the set

$$
\mathbf{E}\left(Q_{0}{ }^{\dagger}\right) Q_{0}{ }^{T}=\mathbf{E}\left(1^{\dagger}\right) Q_{0}{ }^{T} .
$$

By $(\beta 3)$ and the fact that ${Q_{0}}{ }^{T}$ also is a symmetry transformation, $\mathbf{E}\left(1^{\dagger}\right) Q_{0}{ }^{T}$ is a subset of $\mathbf{E}\left(1^{\dagger}\right)$, and $G^{\top} Q_{0}{ }^{T}$ is then a continuation of $1^{\dagger}$ which remains in $\mathbf{E}\left(1^{\dagger}\right)$. The last formula for $\Pi_{0}$ and the definitions of the elastic response $\Pi^{*}$ and the symmetry transformations $Q_{0}$ then yield the relation

$$
\Pi_{0}(A)=\Pi^{*}\left(A, Q_{0}{ }^{\dagger}\right)=\Pi\left(G^{r}\right)=\Pi\left(G^{r} Q_{0}{ }^{T}\right)=\Pi^{*}\left(A Q_{0}{ }^{T}, 1^{\dagger}\right),
$$

and, since $1^{\dagger}$ is a permanent deformation history corresponding to itself, this relation and (P3) tell us that

$$
\Pi_{0}(A)=\Pi^{*}\left(A Q_{0}{ }^{T}, 1^{\dagger}\right)=\Pi_{0}\left(A Q_{0}{ }^{T}\right)
$$

whenever $Q_{0}$ is a symmetry transformation and $A$ is sufficiently close to 1 . Of course, $Q_{0}$ may be replaced by $Q_{0}{ }^{T}$ throughout the proof and hence

$$
\Pi_{0}(A)=\Pi_{0}\left(A Q_{0}\right)
$$

for $Q_{0}$ and $A$ as described above.

It is now possible to state and prove the following proposition.

Given a material with elastic range, a history $F^{t}$, and a permanent deformation history $F_{p}{ }^{t}$ corresponding to $F^{t}$, it follows that $Q_{0} F_{p}{ }^{t}$ is also a permanent deformation history corresponding to $F^{t}$ whenever $Q_{0}$ is a symmetry transformation of the material. In particular, if the material is isotropic, then for every proper orthogonal $Q_{0}$ the history $Q_{0} F_{p}{ }^{t}$ is a permanent deformation history corresponding to $\mathrm{F}^{t}$.

Thus, the existence of many symmetry transformations of the material leads to many possibilities for the permanent deformation. 
The proposition can be established simply by showing that if the conditions (P1), (P2) and (P3) are valid for $F_{p}{ }^{t}$, then they are valid for $Q_{0} F_{p}{ }^{t}$ whenever $Q_{0}$ corresponds to a symmetry transformation.

(P1). By (P1) for the history $F^{t}$, the tensor $F_{p}{ }^{t}(\sigma)$ is in $\mathbf{E}\left(F^{t-\sigma}\right)$; since $Q_{0} F_{p}{ }^{t}(\sigma)$ is in $Q_{0} \mathbf{E}\left(F^{t-\sigma}\right),\left(^{*}\right)$ (Sec. 2) then implies that

$$
Q_{0} F_{p}{ }^{l}(\sigma) \in Q_{0} \mathrm{E}\left(F^{t-\sigma}\right)=\mathbf{E}\left(F^{t-\sigma}\right),
$$

i.e., $Q_{0} F_{p}{ }^{t}$ obeys (P1). (In particular, since $\mathrm{E}\left(F^{t-\sigma}\right)$ is open, the point $Q_{0} F_{p}{ }^{t}(\sigma)$ is not in $\partial \mathrm{E}\left(F^{t-\sigma}\right)$.)

(P2). Suppose that (P2) is not satisfied. There then exists points $A_{1}, A_{2}, \cdots, A_{n}, \cdots$ in $\partial \mathbf{E}\left(F^{t}\right)$ and times $\sigma_{1}, \sigma_{2}, \cdots, \sigma_{n} \cdots$, with $\lim _{n \rightarrow \infty} \sigma_{n}=0$, such that the distance between $A_{n}$ and $Q_{0} F_{p}{ }^{t}\left(\sigma_{n}\right)$ tends to zero as $n$ tends to infinity. However, $Q_{0} F_{p}{ }^{t}\left(\sigma_{n}\right)$ tends to $Q_{0} F_{p}{ }^{t}(0)$ as $n$ tends to infinity, so that the sequence $\left\{A_{n}\right\}$ must tend to the same limit. This is impossible, since the limit of the sequence $\left\{A_{n}\right\}$ must be in $\partial \mathrm{E}\left(F^{t}\right)$ whereas $Q_{0} F_{p}{ }^{t}(0)$ is not in $\partial \mathbf{E}\left(F^{t}\right)$.

(P3). For every $A \in \mathbf{E}\left(F^{t-\sigma}\right)$ which is near $F_{p}{ }^{t}(\sigma), \Pi^{*}\left(A, F^{t-\sigma}\right)=\Pi_{0}\left(A\left(F_{p}{ }^{t}(\sigma)\right)^{-1}\right)$ since $F_{p}{ }^{t}$ is a permanent deformation corresponding to $F^{t}$ and (P3) holds for it. However, for each $Q_{0}$ in the symmetry group of the given material, the preliminary remark implies that

$$
\Pi_{0}\left(A\left(F_{p}{ }^{t}(\sigma)\right)^{-1}\right)=\Pi_{0}\left(A\left(F_{p}{ }^{t}(\sigma)\right)^{-1} Q_{0}{ }^{T}\right)=\Pi_{0}\left(A\left[Q_{0} F_{p}{ }^{t}(\sigma)\right]^{-1}\right),
$$

and this relation holds for all $A$ in $\mathbf{E}\left(F^{t-\sigma}\right)$ close to $F_{p}{ }^{t}(\sigma)$. Hence, for all such $A$ and for all $\sigma \geq 0$

$$
\Pi^{*}\left(A, F^{t-\sigma}\right)=\Pi_{0}\left(A\left[Q_{0} F_{p}{ }^{t}(\sigma)\right]^{-1}\right] .
$$

This argument establishes (P3).

4. Elastic deformations and the non-uniqueness of elastic rotations. Given a history $F^{t}$ and a corresponding permanent deformation history $F_{p}{ }^{t}$, we define for every $\sigma \geq 0$

$$
F_{e}{ }^{t}(\sigma)=F^{t}(\sigma)\left(F_{p}{ }^{t}(\sigma)\right)^{-1}
$$

$F_{e}{ }^{t}$ is called an elastic deformation history corresponding to $F^{t}$. Thus the relation

$$
F^{t}(\sigma)=F_{e}{ }^{t}(\sigma) F_{p}{ }^{t}(\sigma)
$$

holds for all $\sigma \geq 0$. Since, for each symmetry transformation $Q_{0}$, the history $Q_{0} F_{p}{ }^{t}$ also is a permanent deformation history corresponding to $F^{t}$, it follows that for each $Q_{0}$ the history $F_{e}{ }_{e} Q_{0}{ }^{T}$ is an elastic deformation history corresponding to $F^{t}$ whenever $F_{e}{ }^{t}$ is an elastic deformation history. In particular, for an isotropic material the histories $F_{e}{ }_{e}{ } Q_{0}{ }^{T}$ are, for every choice of proper orthogonal $Q_{0}$, elastic deformation histories corresponding to $F^{t}$.

These remarks suffice to establish the non-uniqueness of elastic rotations. In fact, one can write the polar decomposition $F_{e}{ }^{t}=V_{e}{ }^{t} R_{e}{ }^{t}$ for any elastic deformation history $F_{e}{ }^{t}$; thus each orthogonal tensor $Q_{0}$ in the symmetry group gives rise to an elastic deformation history

$$
F_{e}{ }^{t} Q_{0}{ }^{T}=V_{e}{ }^{t}\left(R_{e}{ }^{t} Q_{0}{ }^{T}\right)
$$

having the same stretch $V_{e}^{t}$ but different elastic rotation. 


\section{References}

[1] D. R. Owen, Arch. Rational Mech. Anal. 37, 85 (1970)

[2] E. H. Lee, J. Appl. Mech. 36, 1 (1969)

[3] J. Mandel, C. R. Acad. Sci. Paris 272, Ser. A., 276 (1971)

[4] J. R. Willis, J. Mech. Phys. Solids 17, 359 (1969)

[5] C. Truesdell and W. Noll, The non-linear field theories of mechanics, in Handbuch der Physik III/2, edited by S. Flugge, Berlin-Gottingen-Heidelberg, Springer, 1965

[6] D. R. Owen, Department of Mathematics Technical Report 72-1, January, 1972, Carnegie-Mellon University

Note added in proof: M. Silhavy has kindly pointed out to me that the condition ( $\beta 2)$ actually follows from the priniciple of material frame indifference. Accordingly, beyond frame indifference one need only assume that ( $\beta 3$ ) and the condition on $1^{\dagger}$ at the end of Sec. 2 hold in order to establish the results in this note. 